\section{Fetal surgery}

The earliest operations on the human fetus were destructive in nature to ensure delivery and to protect maternal life. Intrauterine intervention for the benefit of the fetus is of fairly recent origin.

Recently there has been interest in the possibility that certain congenital abnormalities may be corrected in utero. Such methods of treatment have theoretical advantages and have been rendered feasible by the development of techniques of early diagnosis and assessment-such as high resolution ultrasound scanning and fetoscopy. However, only a few disorders are amenable to such treatment. The main conditions of interest are urinary tract outlet obstruction, congenital hydrocephalus, and congenital diaphragmatic hernia.

\section{Urinary tract outlet obstruction}

Posterior urethral valves or urethral stenosis may cause gross bladder distension, often complicated by hydronephrosis. If this occurs early and is severe it may result in the 'prune belly' syndrome. ${ }^{1}$ As with renal agenesis, oligohydramnios is a diagnostic pointer. An ultrasonic diagnosis may be confirmed by injection of contrast medium into the fetal bladder and severely affected cases are visible before 22 weeks. Frequently bladder outlet obstruction has a poor outlook, the kidneys showing irreversible damage at birth. However, as this anomaly is usually an isolated entity, antenatal decompression may be life saving; indeed naturally occurring decompression via a patent urachus may occasionally lead to survival.

A technique for percutaneous suprapubic catheterisation of the fetal bladder has been developed so that the urine drains continuously into the amniotic cavity. ${ }^{2}$ It was used at 32 weeks to decompress the urinary tract of one of twins. The infant born 2 weeks later had normal pulmonary and renal function. The same workers performed bilateral ureterostomy at hysterotomy in the 21 st week of pregnancy on a fetus with bilateral hydronephrosis due to lower urinary tract obstruction. The pregnancy thereafter continued until 35 weeks when the mother was delivered by caesarean section because of the onset of premature labour. The infant had mild facial deformities, limb contractures, and a small chest, although the ureterostomies were functioning. The baby died of severe pulmonary hypoplasia and the kidneys showed irreversible dysplasia. $^{3}$
This technical tour-de-force was evaluated by Eidelman $^{4}$ who pointed out that the unhappy result was not entirely surprising as renal dysplasia occurring secondary to obstruction before the 20th week is usually irreversible and that long-standing oligohydramnios of at least 4-6 weeks, and beginning before 24-26 weeks, causes severe pulmonary hypoplasia. He felt that if oligohydramnios does not occur, fetal surgery is not indicated and the alternative of early delivery should be advised. However, it could be argued that waiting for the onset of oligohydramnios would be the same as waiting for renal failure which earlier decompression might have prevented.

\section{Congenital hydrocephalus}

Early in the second trimester the cerebral mantle is thin and the lateral ventricles and choroid plexus are large and easily seen on ultrasonic scanning. The diagnosis of hydrocephalus due to defective embriogenesis should be possible before 20 weeks' gestational age. From about the 29th week progressive ossification of the fetal skull reduces its elasticity, intracranial pressure increases, and brain under-development occurs.

Monkeys made hydrocephalic in utero by the teratogen triamcinolone acetonide were treated in utero by means of a vent known by the acronym HAVIT (hydrocephalic antenatal vent for intrauterine treatment). This steel device, of about the same size and proportions as a bicycle tyrie valve, was inserted into the anterior horn of the lateral ventricle at hysterotomy and functioned as a one-way valve from the ventricle to the amniotic cavity. These animals failed to develop progressive hydrocephalus and were neurologically nearly normal after birth. 5

In the human, a case has been reported of a patient diagnosed as carrying a hydrocephalic fetus at 24 weeks of pregnancy. Serial percutaneous cephalocenteses guided by ultrasound were performed at $25 \frac{1}{2}, 27 \frac{1}{2}, 29,30$, and $31 \frac{1}{2}$ weeks. Initially $40 \mathrm{ml}$ increasing to $180 \mathrm{ml}$ of cerebrospinal fluid was aspirated. Delivery was by caesarean section at 34 weeks when a permanent ventriculo-peritoneal shunt was inserted. The infant did not develop hydrocephalus although it later had seizures and was found to be suffering from Becker's muscular dystrophy. ${ }^{6}$

More recently a ventriculo-amniotic silicone 
rubber valve was implanted by transuterine puncture in a 24-week fetus, with X-linked hydrocephalus and aqueductal stenosis. The head grew normally until 32 weeks when the valve failed. The infant, delivered at 34 weeks, was fitted with a ventriculo-peritoneal shunt. At age 3 months the infant was showing progressive motor and social development. ${ }^{7}$

\section{Congenital diaphragmatic hernia}

Because of pulmonary hypoplasia, infants born with congenital diaphragmatic hernia have a high mortality rate. Pulmonary hypoplasia results in pulmonary hypertension with pre- and post-ductal shunting, due to the inability of the newborn to expand the lungs and establish an adequate pulmonary vascular circulation. ${ }^{8}$ Survival probably depends more on the degree of pulmonary development at birth than on the quality of neonatal care. The Fetal Treatment Program group in San Francisco, using the fetal lamb model, has shown that pulmonary hypoplasia is a consequence of in utero compression by herniated viscera. ${ }^{9}$ On the basis of experimental findings they suggested that the lung made hypoplastic by congenital diaphragmatic hernia might grow and develop if the compression was relieved. The work has now developed to the stage where they are able to create a diaphragmatic hernia early in the third trimester, cause pulmonary hypoplasia, repair the hernia midway through the third trimester, and demonstrate improved lung development with neonatal survival. ${ }^{10}$ The reduction of the hernia, however, requires the performance of a relieving abdominoplasty to prevent a rise of intra-abdominal pressure, which would otherwise occur and which can lethally reduce the umbilical vein blood flow. This technique has not yet been applied to man.

The main advantage of fetal surgery is that early intervention may prevent permanent damage to vital organs. Healing in utero is rapid and the umbilical circulation provides nutritive and respiratory needs without outside assistance. Potential advantages for the future may lie in the poor fetal immune surveillance system which may facilitate procedures such as the antenatal closure of the neural antrum by allogeneic bone grafting without risk of rejection, and application may even be found for the surprising observation in the non-human primate fetus in whom early severance of some fingers was followed by complete regeneration of the amputated digits. ${ }^{11}$

The primate uterus is a very sensitive organ unlike that of the sheep. Suppression of uterine activity is difficult and requires the use of $\beta$ mimetics and potent prostaglandin synthetase inhibitors, ${ }^{12}$ drugs which may adversely affect the fetus. There is also the ethical dilemma of treating a non-viable fetus as a patient in its own right and one must also consider the mother's interest as it is her body that is the site of treatment. It is also important to distinguish between infants who might benefit from intrauterine surgery and those who would be better transferred to a regional referral centre for optimal neonatal care.

\section{References}

1 Pagon $R$ A, Smith D W, Shepard T H. Urethral obstruction malformation complex: a cause of abdominal muscle deficiency and the 'prune belly'. $J$ Pediatr 1979; 94: 900-6.

2 Golbus M S, Harrison M R, Filly R A, Callen P W, Katz M. In utero treatment of urinary tract obstruction. Am J Obstet Gynecol 1982; 142: 383-8.

3 Harrison M R, Golbus M S, Filly R A, et al. Fetal surgery for congenital hydronephrosis. $N$ Engl J Med 1982; 306: 591-3.

4 Eidelman A I. Editorial. Obstet Anesthesia Dig 1982; 2: 79.

5 Michejda M, Hodgen G D. In utero diagnosis and treatment of non-human primate fetal skeletal anomalies. 1. Hydrocephalus. $J A M A$ 1981; 246: 1093-7.

6 Birnholz J C, Frigoletto F D. Antenatal treatment of hydrocephalus. $N$ Engl J Med 1981; 304: 1021-3.

7 Clewell W H, Johnson M L, Meier P R, et al. A surgical approach to the treatment of fetal hydrocephalus. $N$ Engl J Med 1982; 306: 1320-5.

8 Harrison M R, Bjordal R I, Langmark F, Knutrud $O$. Congenital diaphragmatic hernia: the hidden mortality. $J$ Pediatr Surg 1978; 13: 227-30.

- Harrison M R, Jester J A, Ross N A. Correction of congenital diaphragmatic hernia in utero. 1. The model: intrathoracic balloon produces fatal pulmonary hypoplasia. Surgery 1980; 88: 174-82.

10 Harrison M R, Ross N A, de Lorimer A A. Correction of congenital diaphragmatic hernia in utero. III. Development of a successful surgical technique using abdominoplasty to avoid compromise of umbilical blood flow. J Pediatr Surg 1981 ; 16: 934-42.

11 Hodgen G D. Antenatal diagnosis and treatment of fetal skeletal malformations. JAMA 1981 ; 246: 1079-83.

12 Harrison M R, Anderson J, Rosen M A, Ross N A, Hendrickx A G. Fetal surgery in the primate. 1 . Anesthetic, surgical, and tocolytic management to maximise fetal-neonatal survival. J Pediatr Surg 1982; 17: 115-22.

J F Pearson

Department of Obstetrics and Gynaecology, Welsh National School of Medicine, Heath Park, Cardiff CF4 4 XN 